\title{
Doing und undoing gender in Interaktionen: Analyse der videogestützten Beobachtung
}

\author{
Franziska Vogt
}

\subsection{Interaktionen vermitteln die Bedeutung von Geschlecht}

Die Interaktion zwischen Kinderbetreuenden und Kindern ist wesentliches Qualitätsmerkmal für Betreuung und Bildung in Institutionen der frühen Kindheit (Mortensen und Barnett 2015; NICHD 2002). Entsprechend fokussieren Weiterbildungen darauf, Wege der Professionalisierung zu finden, um die Interaktionsqualität zu verbessern (Egert und Dederer 2018). Die Interaktionsqualität in Kitas wird in Studien aus Deutschland (von Suchodoletz et al. 2014) als niedrig bis moderat und in der Schweiz (Perren et al. 2016) als moderat bis gut eingeschätzt. Während Interaktionsqualität zur Förderung der Kinder in Bezug auf verschiedene sozioemotionale und kognitive Aspekte als relevant angesehen wird, ist auch davon auszugehen, dass die Interaktionsqualität in Bezug auf geschlechterreflektierte Pädagogik von Relevanz ist. Dies umso mehr, da Beobachtungsstudien zur Interaktion im Kita-Alltag aufzeigen, dass in Interaktionen zwischen Kinderbetreuenden und Kindern Geschlechtsstereotype verstärkt werden (Chick et al. 2002).

Dieses Kapitel ist eine adaptierte und erweiterte Fassung des Beitrags, der 2015 in der Schweizerischen Zeitschrift für Bildungswissenschaften erschienen ist: Vogt, F., Nentwich, J. \& Tennhoff, W. (2015). Doing und Undoing Gender in Kitas: Eine Videostudie $\mathrm{zu}$ den Interaktionen von Kinderbetreuenden mit Kindern. Schweizerische Zeitschrift für Bildungswissenschaften, 37(2), 227-247. https://doi.org/10.24452/sjer.37.2.4951

F. Vogt $(\bowtie)$

Pädagogische Hochschule St. Gallen, St.Gallen, Schweiz

E-Mail: franziska.vogt@phsg.ch 
In der Studie von Chick et al. (2002) wurden Kreissequenzen in einer Kita in den USA beobachtet. Die Codierungen erfassten ,gender neutral“, „gender stereotyped“ oder „breaking barriers“, zum Beispiel wenn Erwartungen geäuBert wurden, die den Geschlechterstereotypen widersprechen (Chick et al. 2002, S. 150). Anschließend beschrieben sie die Beobachtungen, die als ,gender stereotyped“ und als „breaking barriers" kategorisiert wurden; über die Kategorie „gender neutral“ finden sich im Beitrag nur wenige Hinweise. Themen der Verstärkung von Geschlechterstereotype zeigten sich wie folgt: Jungen erhielten mehr Aufmerksamkeit und verfügten über mehr Kontrolle in der Kreissequenz; Mädchen erhielten Lob für ihre Kleider, ihr Haar und ihr helfendes Verhalten, während Jungen Bemerkungen zu ihrer Größe und ihrer körperlichen Stärke erhielten; die Kinder wurden sprachlich geschlechterdifferenzierend angesprochen, zum Beispiel Mädchen als „Schätzchen“ und Jungen als „Kumpel“; die Kinder selbst wählten geschlechterstereotype Spielzeuge und Aktivitäten; und es wurden ihnen geschlechterstereotype Vorschläge von den Kinderbetreuenden gemacht. Durchbrechen von Geschlechterstereotypen wurde ebenfalls beobachtet: Mädchen erhielten Unterstützung für sportliche Interessen sowie für geschlechtsuntypische und karriereorientierte Berufswünsche und Jungen wurden positiv bestärkt, wenn sie sich traditionell weiblichen Aktivitäten zuwandten. Auch forderten und förderten die Kinderbetreuenden mit ihrer Gruppenführung für Mädchen wie Jungen die gleichen sozialen Kompetenzen (Chick et al. 2002).

Die experimentelle Studie von Brandes et al. (2013) zeigte auf, dass die Gestaltung der Interaktion weniger vom Geschlecht der Kinderbetreuenden, sondern eher vom Geschlecht der Kinder beeinflusst ist. Den Kinderbetreuenden standen für eine 20-minüitige Interaktion mit einem Kind je zwei Materialkoffer zum Basteln und Werken zur Verfügung. Die Interaktion wurde videografiert und anschließend mit Ratings eingeschätzt. Während sich nur wenige Unterschiede zwischen den Männern und Frauen zeigten, veränderte sich die Situation jedoch je nachdem, ob in der Sequenz mit einem Mädchen oder einem Jungen interagiert wurde. So sprachen Kinderbetreuende mit Jungen eher sachlich-funktional und mit Mädchen eher über die Beziehung oder Persönliches (Brandes et al. 2013, S. 40) und boten jeweils auch unterschiedliche Materialien an. In diesem kontrollierten Experiment zeigte sich eine differenzielle Gestaltung der Interaktion durch die Kinderbetreuenden abhängig vom Geschlecht des Kindes sowie in Übereinstimmung mit den Geschlechterstereotypen. In Anwendung des Verständnisses von doing und undoing gender nach Deutsch (2007) zeigt die Gestaltung der Interaktion durch die Kinderbetreuenden ein doing gender, sie geht von Geschlechterdifferenz aus und verstärkt diese. Während die Studie von Brandes 
et al. (2013) zunächst das Ziel verfolgte zu untersuchen, ob Männer „etwas anderes" in die Kita einbringen, zeigen die Befunde, dass die Herausforderungen für Kinderbetreuende eher darin liegen, Geschlechterstereotype nicht zu verstärken.

Für die Erforschung der Konstruktion von Geschlecht in Interaktionen in Kitas ist die Dynamik der Geschlechterkonstruktion und die Kontextgebundenheit wichtig. Für die Schule zeigten die Arbeiten von Faulstich-Wieland, Weber und Willems (2004) sowie Breidenstein und Kelle (2003) beispielhaft auf, wie diese Prozesse der Geschlechterkonstruktion dynamisch in spezifische Kontexte eingebettet sind. Für die frühe Kindheit eignet sich der Blick auf das Spiel der Kinder besonders gut, um den dynamischen Prozess der Geschlechterkonstruktion zu fassen. Papadopoulou (2012) betont die Bedeutung des Rollenspiels der Kinder als Aneignung und Interpretation des kulturellen Kontextes. Gildemeister und Robert (2008) beschreiben den Kindergarten als Lebensphase der „rigiden Differenzierungen“, auch im Hinblick auf das Spiel der Kinder im Kindergarten. Kubandt (2020) analysiert kindliche Prozesse des doing und undoing gender im Spiel der Kinder untereinander. Sie beschreibt die Aushandlungen der Rollen im Rollenspiel insbesondere im von den Kindern so bezeichneten Mutter-Vater-KindSpiel und die Aufteilung in Gruppen für ein Bewegungsspiel. Die ethnografisch beobachteten Peer-Interaktionen der Kinder im Spiel zeigen beides, doing und undoing gender. Hyun und Choi (2004) zeigen, dass Kinder in Bezug auf das Spiel eine Vorstellung der Geschlechterstereotypen haben, gleichzeitig aber auch in ihr Spiel einbeziehen, wie diese verändert und umgedeutet werden könnten.

Für die Erforschung der Konstruktion von Geschlecht im Kita-Alltag kann demnach davon ausgegangen werden, dass Interaktionen zwischen Kinderbetreuenden und Kindern relevant sind, diese häufig Geschlechterstereotype transportieren und damit als doing gender verstanden werden können, zugleich aber auch, dass undoing gender im Sinn der Betonung nichtstereotyper bzw. die Gleichheit betonender Verhaltensweisen eine Rolle spielt. Geschlechterdifferenz erscheint hinsichtlich des Geschlechts der agierenden Kinderbetreuenden nicht relevant zu sein, jedoch in Bezug auf das Geschlecht des Kindes. Zudem spielt die Interaktion für die Konstruktion von Geschlecht im Kita-Alltag eine wichtige Rolle, ganz besonders im freien Spiel.

\subsection{Doing und undoing gender, Dramatisierung und Dethematisierung}

Für die Analyse der Interaktionen schlagen wir vor, die Konzepte des doing und undoing gender mit Dramatisierung und Dethematisierung zu kombinieren und 
auf der Mikroebene des Interaktionsverlaufs systematisch anzuwenden. Die Analyse der Interaktionen zwischen Kinderbetreuenden und Kindern basiert auf dem Verständnis von Geschlecht als einer fluiden kulturellen Ressource, die in Interaktionen episodisch konstruiert wird (Hirschauer 1994; Deutsch 2007) und derer sich Kinder und Betreuende situationsabhängig bedienen oder auch nicht. Nentwich und Kelan (2014) zeigen auf, dass die Wechsel zwischen doing und undoing schnell und fluide erfolgen. Eine Handlung kann situativ als doing gender interpretiert werden, dann aber zum undoing gender werden. Demzufolge ist es für die Analyse von doing und undoing gender in Interaktionen zwischen Kinderbetreuenden und Kindern wichtig, den Verlauf der Handlung situativ und auf der Mikroebene in kleinen Schritten zu analysieren.

In jeglicher Interaktion wird jeweils die Sexkategorie, also die Zugehörigkeit zu einem Geschlecht, erfasst. Zudem besteht die Möglichkeit, entlang dieser Kategorisierung zur Rechenschaft (,accountability“) gezogen werden zu können (Hollander 2013; West und Zimmermann 2009). Kinder in der Kita sind jeweils als Jungen oder Mädchen erkennbar und ihre Handlungen werden im Wissen um diese Kategorisierung erfasst. Deutsch (2007) weist auf die Notwendigkeit hin, Handlungen auch hinsichtlich ihrer Übereinstimmung mit den mit der jeweiligen Sexkategorie verbundenen Erwartungen in Abgleich zu bringen. So kann in jeder Interaktion Geschlechterdifferenz betont oder aber herausgefordert werden.

Mit Deutsch (2007) kann doing gender als die Betonung der Geschlechterdifferenz gefasst werden, die in der Binarität der Zweigeschlechtlichkeit gefangen ist (Kelan 2010). Dem gegenüber wird undoing gender nach Deutsch (2007) als Betonung von Geschlechtergleichheit verstanden, wie auch als Umschrift der Normen und als ein Stören der Unterscheidungspraktiken (Butler 2004).

Die Betonung der Differenz erfolgt durch bestimmte Tätigkeiten, Gesten und Symbole, die im Alltag als Genderismen zur Verfügung stehen (Goffman 1994). Genderismen bezeichnen die institutionalisierten Arrangements der Geschlechter, wie zum Beispiel die nach Geschlechtern differenzierte Berufswahl, Arbeitsteilung oder auch nach Geschlechtern trennende räumliche Einrichtungen. Für die Analyse in Kitas sind entsprechend die Geschlechtercodierungen bei Spielzeugen besonders relevant (Gildemeister und Robert 2008; Rendtdorff 2011). Ebenfalls werden Geschlechtsstereotype, verstanden als kulturell-symbolische Ressourcen (Pickering 2001), durch die in Alltagshandlungen Geschlecht relevant gemacht werden kann, einbezogen. Auch wenn viele Personen heutzutage die in Geschlechterstereotypen transportierte binäre Unterschiedlichkeit der Geschlechter nicht zwingend als leitende Norm akzeptieren, stellen Geschlechterstereotype nach wie vor stabile Wissensbestände dar. 
Für die Analyse der Interaktionen in der Kita schlagen wir vor diesem Hintergrund vor, doing gender für das Betonen von Geschlechterdifferenz in einer Handlung entlang geltender normativer Erwartungen an ein bestimmtes Geschlecht, ausgedrückt durch den Verweis auf Genderismen oder Geschlechterstereotype, und undoing gender für solche Handlungen, die die Geschlechtergleichheit betonen oder die Genderismen und Geschlechterstereotype infrage stellen, stören oder umdeuten, zu verwenden. Als Beispiel: Wenn ein Junge mit Autos spielt, würde dies als dem Geschlechterstereotyp entsprechend eingeschätzt und als doing gender, wird ein Mädchen dabei beobachtet, würde dies als dem Stereotyp widersprechend und als undoing gender interpretiert.

Aufbauend auf Goffman (1994) und insbesondere Faulstich-Wieland et al. (2004) und ihrer Ausarbeitung des Konzepts der Dramatisierung von Geschlecht im Schulkontext, wird einbezogen, dass Geschlecht in der Dramaturgie einer Interaktion dramatisiert, hervorgehoben, in den Fokus gestellt werden kann. Ebenso, wie Hirschauer (1994) betont, kann Geschlecht nicht nur dramatisiert, sondern auch ruhen gelassen werden. Zwar steht das Wissen über Geschlechterdifferenz als Ressource für (un)doing gender in allen Situationen zur Verfügung, es kann aber situativ auch ruhen gelassen werden. Hirschauer (1994) verwendet den Begriff der Dethematisierung für ein solches Ruhenlassen. Westheuser (2015, S. 112) unterstreicht in seiner Auseinandersetzung mit den Begrifflichkeiten von Hirschauer, dass Dethematisierung nicht unbedingt undoing gender bedeutet: „Eine ausbleibende Dramatisierung von Geschlecht kann auch dafür stehen, dass Geschlecht als Kategorisierungsprinzip vorausgesetzt und wirksam ist. [...] Dethematisierung von Geschlecht steht auch hier eher für das Funktionieren der Unterscheidung, ihre Offensichtlichkeit und Banalität, statt für ein Aussetzen ihrer Wirksamkeit“. Dethematisierung und Dramatisierung werden als eine zweite Analysedimension der Interaktionen herangezogen.

Für die Analyse der Interaktionen soll darum nicht nur doing und undoing gender unterschieden werden, sondern auch im Interaktionsverlauf analysiert werden, ob eine Dramatisierung oder aber Dethematisierung erfolgt. Alle Kombinationen dieser Dimensionen sind möglich. Beispielsweise kann undoing gender dramatisiert werden, indem beispielsweise zu einem Jungen, der mit lackierten Fingernägeln in die Kita kommt, gesagt wird: „Schaut mal, wie schön farbig lackierte Fingernägel Kevin hat, toll!“.

Um die schnellen, situativen Wechsel der Geschlechterkonstruktion in Interaktionen zu erfassen, wird der Interaktionsverlauf auf der Mikroebene in Bezug auf zwei Dimensionen untersucht: doing und undoing gender sowie Dramatisierung und Dethematisierung. In den Interaktionen zwischen Kinderbetreuenden und Kindern soll demnach erstens berücksichtigt werden können, wie Geschlecht 
als Ressource genutzt und ob Geschlechterdifferenz in Übereinstimmung mit stereotypen Erwartungen betont wird (doing gender) oder diesem Wissen widersprochen und Geschlechtergleichheit betont wird (undoing gender). In den Interaktionen soll zweitens unterschieden werden, ob das doing und undoing gender im weiteren Verlauf dramatisiert oder aber dethematisiert wird.

\subsection{Videogestützte Analyse der Interaktionen}

Um die Relevanz von Geschlecht in den alltäglichen Interaktionen in den Kitas zu untersuchen, wurde ein ethnografisches, videogestütztes Vorgehen gewählt. Im Folgenden wird zunächst das Sample für die videogestützte Ethnografie und danach die Erhebung der Daten und das Vorgehen der Analyse dargestellt. Ziel dieser Analyse ist, doing und undoing gender in den Alltagsinteraktionen zwischen Kinderbetreuenden und Kindern zu untersuchen und dabei die Möglichkeiten von Dramatisierung und Dethematisierung einzubeziehen.

\subsubsection{Stichprobe}

Ausgehend von den Interviews mit Kitaleitungen und den Raumanalysen in 20 Kitas wurden vier Kitas ausgewählt, die mindestens einen ausgebildeten männlichen Kinderbetreuer im Team hatten und sich durch unterschiedliche Teamzusammensetzungen, pädagogische Konzepte und Raumgestaltung auszeichneten. Mit diesem kontrastierenden, theoretischen Sampling sollte gewährleistet werden, dass für die ethnografische Beobachtung und Videobeobachtung möglichst unterschiedliche Fälle zur Verfügung stehen. In den Kitas wurden diejenigen Gruppen ausgewählt, in denen je mindestens eine weibliche und eine männliche Betreuungsperson arbeitete. Die Mitarbeitenden und die Eltern der Kinder der ausgewählten Gruppen wurden über die Studie informiert, für die Videoaufnahmen wurden Einverständniserklärungen eingeholt. Kinder, deren Eltern keine Einwilligung zur videogestützten Beobachtung gaben, wurden während der Videografierung in einer anderen Gruppe der Kita betreut. 


\subsubsection{Design Videostudie}

Jede Kita wurde während vier ganzen Tagen besucht, in der Regel je zwei Tage hintereinander über zwei Wochen, bei einer Kita konnten lediglich drei Beobachtungstage stattfinden. Neben den ethnografischen Beobachtungen, die in Protokollen festgehalten wurden, wurde so oft wie möglich mit einer Videokamera das Geschehen aufgenommen. Dabei wurde abwechslungsweise ein Kinderbetreuer oder eine Kinderbetreuerin mit einem Funkmikrofon ausgerüstet und die Kamera folgte deren Tätigkeit möglichst unauffällig aus der Distanz. Das so erhobene Videomaterial im Umfang von 50 Stunden wurde mittels der Software Atlas.ti vollständig analysiert. Für die Analyse der Interaktionen wurden mithilfe des Eventsamplings (Ostrov und Hart 2013) für die Fragestellung relevante und für die Kita typische Sequenzen ausgewählt. Diese Auswahl umfasst kurze, etwa einminütige Interaktionen bis zu längeren, etwa zehnminütigen Sequenzen. Für die nicht ausgewählten Sequenzen wurde ein Memo verfasst, das zusammenfassend darstellt, was auf diesen Filmteilen zu sehen ist und warum diese nicht für eine nähere Analyse ausgewählt wurden.

Jede ausgewählte Sequenz wurde in Bezug auf vier Aspekte kategorisiert: (i) gender (ii) Interaktion (iii) Raum und Material und (iv) Sozialform und Aktivität. Die verwendeten Kategorien und die Codierregeln sind in Tab. 4.1 aufgeführt.

Für die Überprüfung der Intersubjektivität der Auswahl der Sequenzen und ihrer Kategorisierung wurden vier Aufnahmen von mindestens je einer halben Stunde von je einer weiteren Forscherin unabhängig codiert. Die Überprüfung zeigte, dass innerhalb des Forschungsteams ein Konsens zur Auswahl von Sequenzen in Bezug auf doing und undoing gender bestand. Die Abgrenzung einzelner Sequenzen war unterschiedlich (zum Beispiel genauer Beginn und Ende der Sequenzen); dieser Aspekt ist für die weitere Analyse jedoch nicht relevant. Die Kategorisierung zu Interaktion, Raum und Material und Sozialform und Aktivität erzielte eine sehr hohe Übereinstimmung. Die Zuteilung der Subkategorien betreffend gender erwies sich als schwierig, da eine große Fluidität im Moment festzustellen war. Darum wurde in der weiteren Analyse neben der Kategorisierung zu doing und undoing gender sowie Dethematisierung immer auch ein Memo zu jeder Sequenz geschrieben. Diese Memos bilden eine erste, interpretative Beschreibung der Sequenz hinsichtlich des stattfindenden (un)doing gender. 
Tab. 4.1 Analysekategorien der Videoanalyse für die gesampelte Sequenzen. Grau hinterlegt die für den Datenkorpus der Interaktionsanalyse im freien Spiel verwendeten (Sub-)Kategorien

\begin{tabular}{|c|c|c|}
\hline Kategorie & Subkategorie & Beschreibung, ggf. Codierregel \\
\hline Gender & & $\begin{array}{l}\text { Einer Sequenz können mehrere Subkategorien zugewiesen } \\
\text { werden. In einem Memo wird zur Sequenz beschrieben, } \\
\text { was als doing/undoing und Dethematisierung interpretiert } \\
\text { wird. Es wird mindestens eine Gender-Subkategorie } \\
\text { vergeben. }\end{array}$ \\
\hline Gender & Doing gender & $\begin{array}{l}\text { Gender wird stereotypisch, differenzbetonend relevant } \\
\text { gemacht, durch Symbolik, Interaktion, Kommunikation } \\
\text { auch nur Handlung oder Raum }\end{array}$ \\
\hline Gender & Undoing gender & $\begin{array}{l}\text { Mit Symbolik, in Interaktion, Kommunikation und } \\
\text { Handlungen werden stereotype Geschlechterbilder } \\
\text { umgedeutet, verändert, unterwandert oder Gleichheit } \\
\text { betont }\end{array}$ \\
\hline Gender & Thematisiert & $\begin{array}{l}\text { Es wird über gender etwas gesagt, dieser Code wird immer } \\
\text { noch mit entweder doing oder undoing doppelt codiert. }\end{array}$ \\
\hline Gender & Dethematisierung & Gender wird ruhen gelassen. \\
\hline Interaktion & & $\begin{array}{l}\text { Es wird immer eine Interaktionssubkategorie vergeben, in } \\
\text { der Regel nur einfach codiert, für neue Interaktionen wird } \\
\text { eine neue Sequenz definiert }\end{array}$ \\
\hline Interaktion & Erwachsene - Erwachsene & $\begin{array}{l}\text { Interaktion Erwachsene - Erwachsene, meistens die } \\
\text { Kinderbetreuenden untereinander }\end{array}$ \\
\hline Interaktion & Kinderbetreuer - Kind & Interaktion männlicher Kinderbetreuer Kind \\
\hline Interaktion & Kinderbetreuerin - Kind & Interaktion weibliche Kinderbetreuerin Kind \\
\hline Interaktion & Forschende - Feld & $\begin{array}{l}\text { Interaktion zwischen Forschenden mit jemandem aus dem } \\
\text { Feld, meistens Kinderbetreuende. Meistens Gespräche } \\
\text { über Pädagogik, Kita-Strukturen, gender (Metaebene) }\end{array}$ \\
\hline Interaktion & Kind - Kind & Interaktion zwischen zwei oder mehreren Kindern \\
\hline Körperkontakt & & $\begin{array}{l}\text { Körperkontakt wird nur codiert, wenn Körperkontakt } \\
\text { zwischen Kinderbetreuenden und Kindern vorkommt. } \\
\text { Körperkontakt wird nicht codiert, wenn er nicht } \\
\text { vorkommt. }\end{array}$ \\
\hline Körperkontakt & diverse & $\begin{array}{l}\text { Wenn Körperkontakt codiert wird, aber die anderen Codes } \\
\text { nicht zutreffen }\end{array}$ \\
\hline Körperkontakt & Hilfe & $\begin{array}{l}\text { Hilfestellungen zum Gehen, Klettern und für } \\
\text { feinmotorische Aktivitäten }\end{array}$ \\
\hline Körperkontakt & Loben & Beispielsweise auf die Schultern klopfen, umarmen \\
\hline Körperkontakt & Pflege & $\begin{array}{l}\text { Pflege, auch An- und Abziehen. Hier wird dann doppelt } \\
\text { codiert mit dem Code Sozialform: Pflege, Hygiene oder } \\
\text { mit Sozialform: Anziehen. }\end{array}$ \\
\hline Körperkontakt & Trösten & Trösten, z. B. über den Kopf streichen, umarmen, halten. \\
\hline Körperkontakt & Zurechtweisen & Zurechtweisen, z. B. zurückbehalten, festhalten \\
\hline Raum/Material & & $\begin{array}{l}\text { Kategorie für Spielorte und Material, muss für jede } \\
\text { Sequenz vergeben werden. Raum diverse dient als Rest- } \\
\text { Code. Einer Sequenz können mehrere Subkategorien } \\
\text { zugewiesen werden. }\end{array}$ \\
\hline Raum/Material & $\begin{array}{l}\text { Autos, Züge, Baustelle } \\
\text { (kleines Rollenspiel, } \\
\text { männlich konnotiert) }\end{array}$ & $\begin{array}{l}\text { Wenn mit männlich konnotiertem Material für das kleine } \\
\text { Rollenspiel gespielt wird, wie Autos, Züge, Bagger }\end{array}$ \\
\hline Raum/Material & Bauecke & Bauecke oder Bauspiel, Bauklötze, Kappla, Lego \\
\hline
\end{tabular}


Tab.4.1 (Fortsetzung)

\begin{tabular}{|c|c|c|}
\hline Kategorie & Subkategorie & Beschreibung, ggf. Codierregel \\
\hline Raum/Material & $\begin{array}{l}\text { diverse andere Räume und } \\
\text { Materialien }\end{array}$ & $\begin{array}{l}\text { Räume/Materialien, die nicht in die vorgegebenen } \\
\text { Kategorien passen, aber die auch nicht so wichtig sind, } \\
\text { dass dafür ein eigener Raumcode geschaffen werden soll. } \\
\text { Da bei jeder Sequenz immer ein Raum/Material Code } \\
\text { vergeben wird, ist dieser Code der 'Rest'-Code }\end{array}$ \\
\hline Raum/Material & draussen & Draussen, Garten, Spielplatz, Wald, unterwegs \\
\hline Raum/Material & $\begin{array}{l}\text { kleines } \\
\text { diverses }\end{array}$ & $\begin{array}{l}\text { Kleines Rollenspiel, das nicht männlich oder weiblich } \\
\text { konnotiert ist }\end{array}$ \\
\hline Raum/Material & Küchen/Puppenecke & $\begin{array}{l}\text { Wenn in der Ecke mit Küche, Tisch, Bettchen und so } \\
\text { weiter gespielt wird und wenn mit Puppen, Puppenwagen } \\
\text { gespielt wird }\end{array}$ \\
\hline Raum/Material & Malen, Basteln, Werken & $\begin{array}{l}\text { Bastelecke oder Werkbank, aber auch wenn Kinder } \\
\text { irgendwo malen, basteln oder werken }\end{array}$ \\
\hline Raum/Material & $\begin{array}{lr}\text { Puppenhaus } & \text { (kleines } \\
\text { Rollenspiel, } & \text { weiblich } \\
\text { konnotiert) } & \end{array}$ & $\begin{array}{l}\text { Wenn mit weiblich konnotiertem Material für das kleine } \\
\text { Rollenspiel gespielt wird, wie Puppenstuben, Little Pet } \\
\text { Shop }\end{array}$ \\
\hline Raum/Material & Ruhebereich & $\begin{array}{l}\text { vor allem für ruhige Ecken (Matratzen, Schleier, Sofa) aber } \\
\text { auch für Schlafzimmer möglich }\end{array}$ \\
\hline Raum/Material & Spielzimmer & $\begin{array}{l}\text { Der gesamte Raum, was nicht eine bestimmte Ecke ist, also } \\
\text { auch inklusive Teppiche in der Mitte des Raumes für } \\
\text { Spielsituationen }\end{array}$ \\
\hline Raum/Material & Tisch & $\begin{array}{l}\text { An den Tischen wird gespielt, auch Puzzle, Brettspiele, } \\
\text { Experimente }\end{array}$ \\
\hline Raum/Material & unstrukturiertes Material & $\begin{array}{l}\text { Mit unstrukturiertem Material, } \\
\text { Tannenzapfen, Holzabfall, Sand }\end{array}$ \\
\hline Raum/Material & $\begin{array}{l}\text { Verkleidung, grosses } \\
\text { Rollenspiel }\end{array}$ & $\begin{array}{l}\text { Spiel mit Verkleidung, grosses Rollenspiel, enthält alles, } \\
\text { also auch die Stöckelschuhe in der Küchenecke (dann } \\
\text { doppelcodiert) aber auch Tierhüte, Polizeiverkleidung, } \\
\text { Schaffnermütze, Tücher (dann doppelcodiert mit } \\
\text { unstrukturiertem Material) }\end{array}$ \\
\hline Sozialform & & $\begin{array}{l}\text { Kategorie für die Sozialform und für die zeitliche } \\
\text { Strukturierung im Tagesablauf, sie verortet die Sequenz im } \\
\text { Kita-Alltag. Sie muss für jede Sequenz vergeben werden, } \\
\text { diverse gilt als Rest-Subkategorie. Einer Sequenz können } \\
\text { mehrere Subkategorien zugewiesen werden. }\end{array}$ \\
\hline Sozialform & An- und Ausziehen & $\begin{array}{l}\text { Phasen in denen Kinder angezogen werden oder sich } \\
\text { anziehen. Wenn Kinderbetreuende mithelfen, gemeinsam } \\
\text { mit Körperkontakt Pflege codieren }\end{array}$ \\
\hline Sozialform & Angeleitet einzeln & $\begin{array}{l}\text { Kinderbetreuerin/Kinderbetreuer leitet ein Kind an, z. B. } \\
\text { beim Spiel, beim Basteln, beim Buchanschauen, beim } \\
\text { Experiment }\end{array}$ \\
\hline Sozialform & Angeleitet Gruppe & $\begin{array}{l}\text { Kinderbetreuerin/Kinderbetreuer macht mit einer Gruppe } \\
\text { von Kindern etwas, z. B. Lied singen, Kreisspiel }\end{array}$ \\
\hline Sozialform & $\begin{array}{l}\text { Aufräumen, Ordnung } \\
\text { herstellen }\end{array}$ & $\begin{array}{l}\text { Kinderbetreuende räumen selber auf oder halten die } \\
\text { Kinder dazu an, aufzuräumen }\end{array}$ \\
\hline Sozialform & Diverses & Sozialform Restkategorie \\
\hline Sozialform & Essen & Mittagessen, gemeinsame Zwischenmahlzeiten \\
\hline Sozialform & $\begin{array}{l}\text { Freies Spiel, freie } \\
\text { Aktivität }\end{array}$ & $\begin{array}{l}\text { Die Aktivität ist nicht von den Kinderbetreuenden initiiert, } \\
\text { wird jedoch möglicherweise von den Kinderbetreuenden }\end{array}$ \\
\hline
\end{tabular}


Tab.4.1 (Fortsetzung)

\begin{tabular}{|l|l|l|}
\hline Kategorie & Subkategorie & Beschreibung, ggf. Codierregel \\
\hline Sozialform & Konfliktsituation & $\begin{array}{l}\text { begleitet, es machen auch verschiedene Kinder } \\
\text { verschiedenes }\end{array}$ \\
\hline Sozialform & $\begin{array}{l}\text { Nur wenn die Konfliktsituation auch ein interessantes } \\
\text { Potenzial für gender hat oder wenn sie länger ist. Nicht } \\
\text { jedes Mal, wenn ein Kind einem andern was wegnimmt, } \\
\text { schlägt }\end{array}$ \\
\hline Sozialform & Pflege, Hygiene & $\begin{array}{l}\text { Händewaschen, Wickeln, wenn Kinderbetreuende } \\
\text { mithelfen gemeinsam mit Körperkontakt Pflege codieren }\end{array}$ \\
\hline
\end{tabular}

\subsubsection{Datenkorpus Videosequenzen}

Für die Analyse der Interaktion zwischen Kinderbetreuenden und Kindern wurden aus allen ausgewählten Sequenzen jene 352 ausgewählt, in denen eine Interaktion zwischen Erwachsenen und Kindern stattfindet. Von diesen Sequenzen wurden wiederum diejenigen 151 ausgewählt, die mit den Subkategorien Sozialform freies Spiel/freie Tätigkeit (unabhängig von Raum und Material) codiert wurden. Um sicherzugehen, dass keine Spielsituationen in den verschiedenen Rollenspielecken übersehen wurde, wurde diese Auswahl noch mit insgesamt zwölf Sequenzen ergänzt, die bei den Räumen bzw. Materialien mit einer der Subkategorien Autos und Züge, Bauecke, kleines Rollenspiel diverse, Puppen/Küchenecke, Puppenhaus, Spielzimmer oder Verkleidung großes Rollenspiel (mit jeweils irgendeiner Sozialform) codiert waren. Die meisten dieser zusätzlichen Sequenzen wurden mit der Sozialform Aufräumen codiert, es sind also Interaktionen zwischen Kinderbetreuenden und Kindern am Ende des Rollenspiels. Auf diese Weise konnten der Fokus auf das freie Spiel sichergestellt und zugleich die Spielorte des Rollenspiels vollständig integriert werden.

Aus diesen insgesamt 163 Sequenzen wurden für die weitere Analyse diejenigen Sequenzen aussortiert, die ausschließlich durch die Kinderbetreuenden initiiert wurden. Das verbleibende Sample von 119 Interaktionen bestand damit ausschließlich aus Interaktionen, die durch eine Handlung oder Äußerung eines Kindes begonnen wurde, auf die dann die Kinderbetreuenden reagierten oder nicht reagierten. Aufgrund dieser Auswahl war es uns möglich, doing und undoing gender in den Interaktionen der Kinderbetreuenden mit den Kindern zu untersuchen. 
Tab.4.2 Datenkorpus für die Analyse

\begin{tabular}{l|l|l|l|l|l}
\hline $\begin{array}{l}\text { Anzahl } \\
\text { Sequenzen }\end{array}$ & $\begin{array}{l}\text { Kinderbetreuer } \\
\text { mit Jungen }\end{array}$ & $\begin{array}{l}\text { Kinderbetreuer } \\
\text { mit Mädchen }\end{array}$ & $\begin{array}{l}\text { Kinderbetreuerin } \\
\text { mit Jungen }\end{array}$ & $\begin{array}{l}\text { Kinderbetreuerin } \\
\text { mit Mädchen }\end{array}$ & $\begin{array}{l}\text { Total } \\
\text { Sequenzen } \\
\text { pro Kita }\end{array}$ \\
\hline Kita Gelb & 19 & 10 & 5 & 0 & 34 \\
\hline Kita Rot & 7 & 4 & 4 & 9 & 24 \\
\hline Kita Grün & 9 & 5 & 2 & 7 & 23 \\
\hline Kita Blau & 7 & 14 & 10 & 7 & 38 \\
\hline Total & 42 & 33 & 21 & 23 & 119 \\
\hline
\end{tabular}

Der so erstellte Datenkorpus umfasst 119 Sequenzen, die sich in Bezug auf die Kitas, das Geschlecht der Kinderbetreuenden und das Geschlecht der Kinder, wie in Tab. 4.2 aufgeführt, verteilen.

Es entfallen insgesamt 63 Sequenzen auf Interaktionen mit Jungen und 56 auf Interaktionen mit Mädchen. Es sind mehr Sequenzen mit Kinderbetreuern ( $\mathrm{n}=$ 75) als mit Kinderbetreuerinnen $(n=44)$ im Datenkorpus. Vor allem in Kita Gelb sind die Sequenzen im Datenkorpus ungleich verteilt. Der Kinderbetreuer in Kita Gelb konnte länger gefilmt werden als die Kinderbetreuerin, da diese zugleich Gruppenleiterin und stellvertretende Kitaleiterin war und deswegen die Gruppe auch zwischendurch verließ.

\subsubsection{Analyse der Interaktionsverläufe}

Für alle 119 ausgewählten Interaktionen haben wir den gesamten Verlauf hinsichtlich des stattfindenden doing und undoing gender, sowie Dramatisierung und Dethematisierung untersucht. Ausgangspunkt der Analyse ist jeweils die beginnende Handlung oder Äußerung des Kindes in der Interaktion. In einem ersten Schritt wurde entschieden, inwieweit in dieser Handlung die Geschlechterdifferenz durch Geschlechterstereotypen betont wird (doing gender) oder diese umgedeutet werden und somit Geschlechtergleichheit betont wird (undoing gender). Die Kategorisierung für doing und undoing gender geschieht in Bezug auf die Erwartungen in Bezug auf das Geschlecht der handelnden Person und des situativen Kontexts (accountability; Deutsch 2007; Hollander 2013). Um Tätigkeiten als dem Geschlechterstereotyp entsprechend oder widersprechend einzuteilen, wurden Stereotypenforschung, Analysen der Geschlechtercodierung bei Spielzeug (Rendtdorff 2011) sowie Aussagen aus den Interviews des Forschungsprojekts 
mit Kitaleitungen, Kinderbetreuerinnen und -betreuern über geschlechtertypische Erwartungen an Buben und Mädchen beigezogen.

Im zweiten Schritt wird die Reaktion oder Nichtreaktion der Kinderbetreuenden analysiert. In der Analyse des Interaktionsverlaufs wird untersucht, wie etwas getan wird. Hier wird zwischen Dramatisierung und Dethematisierung unterschieden. Wird zum Beispiel eine geschlechterdifferenzierende Konnotierung der Situation explizit von den Kinderbetreuenden kommentiert, interpretieren wir dies als Dramatisierung, verläuft sie unbemerkt im Hintergrund, ordnen wir sie als Dethematisierung ein. Ebenso können Umdeutung und Herausforderung der Geschlechterstereotypen in den Vordergrund gestellt werden oder nicht zum Thema gemacht werden.

In Situationen, in denen die Kinderbetreuenden mit Dethematisierung reagieren und Geschlecht nicht weiter relevant gemacht wird, ist der Verlauf dieser einzelnen Interaktion abgeschlossen. Wenn die Kinderbetreuenden mit Dramatisierung auf die Passung oder die fehlende Passung zwischen der Handlung und dem Geschlechterstereotyp, jeweils bezogen auf das Geschlecht des Kindes (Faulstich-Wieland et al. 2004) reagieren, wird diese Reaktion im dritten Schritt wiederum in Bezug auf doing und undoing gender analysiert. Die Kinderbetreuenden haben in der Dramatisierung zwei Möglichkeiten: Sie können mit doing gender die Geschlechterstereotypen betonen und einfordern oder sie können mit undoing gender eine Handlung bestärken oder einfordern, die den stereotypen Erwartungen entgegenläuft. Anhand der Analyseschritte lassen sich sechs mögliche Interaktionsverläufe unterscheiden (Abb. 4.1). Alle Interaktionen wurden entlang dieser zwei bzw. drei Schritte analysiert und aufgrund der sechs möglichen Interaktionsverläufe codiert.

\subsection{Interaktionsverläufe im Spannungsfeld von doing und undoing gender, Dramatisierung und Dethematisierung}

Zur Darstellung der Ergebnisse wird zunächst beschrieben, wie sich die theoretisch entwickelten sechs Interaktionsverläufe im Material zeigen. Dafür wird für jeden Interaktionsverlauf ein Beispiel illustriert. Danach wird die Verteilung der Interaktionsverläufe beschrieben. 


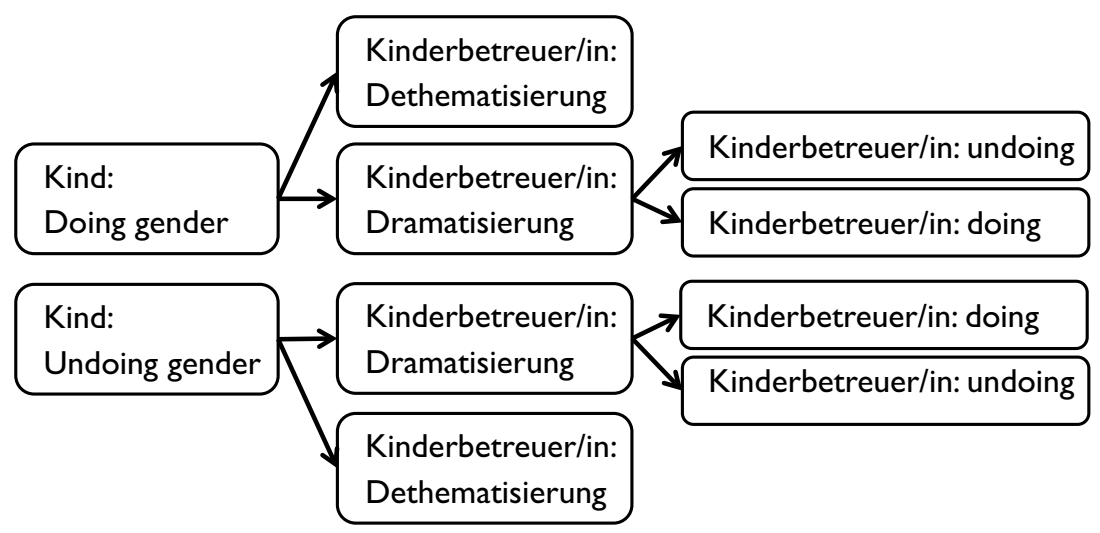

Abb. 4.1 Interaktionsverläufe für die Interaktionen zwischen Kind und Kinderbetreuerin oder -betreuer in Bezug auf (un)doing gender und Dramatisierung bzw. Dethematisierung

\subsubsection{Kind doing gender, Kinderbetreuende Dethematisierung}

Dass ein Kind doing gender zeigt und die Kinderbetreuenden dies nicht beachten und dies so dethematisiert wird, tritt in sechs der Sequenzen dieses Datenkorpus auf. Häufig folgen hier die Kinderbetreuenden dem Spiel der Kinder oder nehmen hieran teil, ohne jedoch die hierin potenziell liegende geschlechtliche Konnotation aufzugreifen. Als Beispiel hier die Interaktion zwischen einem Kinderbetreuer und einem Jungen während der Freispielphase im Wald:

Der Junge schnitzt mit einem Taschenmesser am Stecken. Der Kinderbetreuer erklärt ihm in Worten, wie er das Messer halten soll, wie er schnitzen kann und welche Regeln für das Schnitzen gelten. (Kita Grün)

Mit dem Taschenmesser zu schnitzen, entspricht einer geschlechterstereotypisch männlichen Tätigkeit. Der Kinderbetreuer geht jedoch auf dieses doing gender nicht ein, sondern erklärt die Tätigkeit an sich, wodurch Geschlecht dethematisiert wird. 


\subsubsection{Kind doing gender, Kinderbetreuende Dramatisierung mit doing gender}

In diese Kategorie fallen 34 Sequenzen. Als Beispiel ein Austausch eines Kinderbetreuers mit einer Gruppe Jungen über Werkzeuge:

Ein Bub hält ein Werkzeug in der Hand, der Kinderbetreuer erklärt, was es ist und wofür es gebraucht wird. Die Buben nehmen Werkzeuge aus der Kiste und spielen damit. Der Kinderbetreuer sagt zu einem Buben: „Da kommt der Baumann!“ (Kita Grün)

Die Dramatisierung und doing gender wird hier mit dem Ausdruck „Da kommt der Baumann" sichtbar, eine Anspielung auf die Kinderserie Bob der Baumeister. Das Beispiel zeigt exemplarisch, wie gender in der Spielbegleitung relevant gemacht wird. Dies geschieht häufig durch Zuschreibungen und dem Ausdruck von Erwartungen, was ein Kind ist oder sein könnte. In diesem Fall wird die dem Geschlechterstereotyp entsprechende Aktivität des Jungen, das Bauen, verstärkt, indem dem Jungen die Rolle des Baumanns zugeschrieben wird.

In einem weiteren Beispiel redet die Kinderbetreuerin mit einem Mädchen sehr lange über Mode. Ein hinzukommender Junge wird nicht beachtet und nicht in die Interaktion einbezogen.

Die Kinderbetreuerin sitzt auf einer Bank und hat ein Mädchen auf dem Schoß. Sie trägt ein pinkes T-Shirt, darauf sind Schuhe abgebildet und es gibt einige Applikationen, wie Tüll und Pailletten, dazu einen Schriftzug „Fashion makes me pretty“. Das Mädchen nestelt am T-Shirt der Kinderbetreuerin, sie sagt: „Da sind Schuhe“. Die Kinderbetreuerin präzisiert: „Es sind Ballerinas“. Das Mädchen zählt die Pailletten auf dem T-Shirt. Dann fragt sie, was auf dem T-Shirt steht. Die Kinderbetreuerin übersetzt: „Mode macht mich schön“. (Kita Blau)

Das Beispiel zeigt, wie der sogenannte gender display (Goffman 1994) zur Aktivierung der Kategorie Geschlecht beiträgt und damit doing gender initiieren kann. Gender wird hier dramatisiert: Der Schriftzug wie auch die Kleidung der Betreuerin vermittelt eine stereotypisierende Botschaft, das Mädchen initiiert ein sehr langes Gespräch über das T-Shirt, auf das die Betreuerin fokussiert bleibt. Das Mädchen sitzt insgesamt während 25 min auf dem Schoß der Betreuerin, andere im Raum anwesende Kinder werden nicht beachtet und nicht einbezogen. Die geschlechterstereotypen Inhalte werden durch die genaue Bezeichnung der Schuhe und durch die Übersetzung von „fashion makes me pretty“ dramatisiert. 
Die Aussage „Mode macht mich schön“ wird von der Betreuerin weder diskutiert noch infrage gestellt.

\subsubsection{Kind doing gender, Kinderbetreuende Dramatisierung mit undoing gender}

In dieser Kategorie befinden sich 14 Sequenzen, in denen dem mit stereotypen Erwartungen konformen Verhalten der Kinder mit einem dramatisierenden undoing gender der Betreuenden begegnet wird. Als Beispiel dient die Begleitung des Kinderbetreuers bei einer Aktivität auf einer Leiter:

Ein Mädchen und ein Junge klettern auf einer eigens errichteten Bogenleiter im Spielzimmer. Das Mädchen steigt vorsichtig auf der Leiter nach vorn, um dann hinunterzuklettern. Der Junge ist schon hinuntergeklettert und sagt zum Mädchen: „Hast du Angst?“ Der Kinderbetreuer sagt zum Jungen: „Nein, sie will es anders“. (Kita Rot)

Hier wird eine geschlechtsstereotype Deutung eines Kindes vom Kinderbetreuer thematisiert und umgedeutet und damit dramatisiert. Er greift die Interpretation des Jungen auf, indem er seiner geschlechterstereotypen Interpretation widerspricht und eine alternative Interpretation des vorsichtigen Verhaltens des Mädchens anbietet. Wie Giess-Stüber, Voss und Petry (2003) aufzeigen, gibt es geschlechterdifferenzierende Erwartungen und Hilfestellungen in der frühkindlichen Bewegungsförderung, die unter anderem Ängstlichsein eher mit Mädchen in Verbindung bringen. Doing und undoing gender der Bewegungserziehung in der frühen Kindheit ist jedoch noch kaum untersucht (Gramespacher und Voss 2019).

In dieser Kategorie finden sich auch einige Sequenzen, in denen Jungen aufgefordert werden, nicht wild und laut zu spielen, sondern ruhig zu sein. Die Reaktion auf das wilde Spiel mit der Betonung von Ordnungen, seien es Zeitordnungen oder Raumordnungen, kann als eine Betonung von ,hausfraulicher Weiblichkeit“ interpretiert werden (Rabe-Kleberg 2003; Kap. 5). Nachdem nun drei Verläufe beschrieben wurden, die von einem doing gender des Kindes ausgehen, folgen nun die drei Interaktionsverläufe, bei denen ein undoing gender des Kindes am Anfang steht. 


\subsubsection{Kind undoing gender, Kinderbetreuende Dramatisierung mit doing gender}

In dieser Kategorie befinden sich insgesamt 14 Sequenzen. Als Beispiel dient eine Sequenz, bei der mehrere Jungen in der Puppenecke spielen. In der Puppenecke hat es unter anderem Stöckelschuhe als Requisiten.

Ein Junge sitzt neben dem Kinderbetreuer am Boden und zieht Stöckelschuhe an. Der Kinderbetreuer sagt zu ihm: „Sieht lustig aus. Bist du s'Fräulein?“ Junge: „Nein!“ Kinderbetreuer: „Mit den Stöckelschuhen... Bist du das Mami?“ Junge: „Tim ist das Mami“. (Kita Gelb)

Die Handlung des Jungen, Stöckelschuhe anzuziehen, wird als undoing gender interpretiert. Der Kinderbetreuer weist in seiner Aussage auf die Diskrepanz zwischen Handlung und geschlechtsstereotypen Erwartungen hin und bietet dem Jungen weibliche Rollen, „Fräulein“ und „Mami“, an. Im vorgestellten Interpretationsrahmen kann die Reaktion des Kinderbetreuers als Dramatisierung gelesen werden; der Umstand, dass hier die Nichtpassung von symbolischem Geschlecht der Handlung und Geschlecht des Kindes mit einem weiblichen Rollenangebot versehen wird, qualifiziert sie als doing gender. Die Geschlechterordnung wird mit dem passenden weiblichen Rollenangebot durch den Kinderbetreuer eingefordert.

\subsubsection{Kind undoing gender, Kinderbetreuende Dramatisierung mit undoing gender}

In dieser Kategorie gibt es 40 Sequenzen, in denen die nicht dem Stereotyp entsprechenden Aktivitäten der Kinder durch die Reaktion der Kinderbetreuenden verstärkt werden. Im folgenden Beispiel unterstützt die Kinderbetreuerin das nichtstereotype Rollenspiel eines Mädchens:

Das Mädchen kommt zur Kinderbetreuerin und sagt, dass der Bauhelm kaputt sei. Die Kinderbetreuerin geht mit ihr zur Puppenecke zurück und sagt, es hätte noch einen andern. Sie finden einen Polizeihut, den das Mädchen haben möchte. Der erste Polizeihut ist zu groß, sie nimmt den zweiten und gibt den anderen der Kinderbetreuerin, die ihn auch aufsetzt. Die Kinderbetreuerin hilft dem Mädchen beim Anziehen der Jacke und sagt: „Jetzt bist du eine Polizistin“. (Kita Rot) 
Die Kinderbetreuerin unterstützt das Vorhaben des Mädchens, eine nicht geschlechterstereotype Rolle zu spielen und bietet ihr mit der Zuschreibung „Polizistin“ auch eine Rolle zur Identifikation an.

\subsubsection{Kind undoing gender, Kinderbetreuende Dethematisierung}

Bei diesen Sequenzen reagieren die Kinderbetreuerin oder der Kinderbetreuer nicht auf das vom Kind gezeigte geschlechtsuntypische Verhalten. Insgesamt sind elf Sequenzen in dieser Kategorie. Als Beispiel dient die folgende auf einer im Freien zum Spielen eingerichteten Baustelle stattfindende Interaktion:

Auf der Baustelle sitzt ein kleines Mädchen neben der Kinderbetreuerin und sägt, die Kinderbetreuerin beachtet es nicht, sie redet mit den Jungen, die auch sägen. (Kita Grün)

Das Mädchen beschäftigt sich mit einem Werkzeug, das der geschlechtsstereotypen Erwartung widerspricht und darum als undoing gender interpretiert werden kann. Dies wird jedoch weder kommentiert noch anderweitig besonders beachtet.

Zusammenfassend zeigt sich in den Interaktionsverläufen, dass gender in der Kita in sehr unterschiedlicher Art und Weise relevant gemacht wird. Das Verhalten der Kinder kann den aufgrund der Sexkategorisierung des Kindes bestehenden stereotypen Erwartungen entweder entsprechen oder aber zuwiderlaufen. Diese Unterschiede haben wir doing und undoing gender eingeordnet. Die an der Interaktion beteiligten Kinderbetreuenden können nun auf das gezeigte Verhalten eingehen - oder aber es im Hintergrund ruhen lassen, sie dramatisieren oder dethematisieren. Wenn die Kinderbetreuenden darauf eingehen, dramatisieren, geschieht dies wiederum entweder mit doing oder undoing gender, indem sie Geschlechterdifferenz betonen oder einfordern oder Geschlechtergleichheit sowie das Umdeuten und Stören der Geschlechterordnung unterstreichen. Nachdem wir die verschiedenen Verlaufsmöglichkeiten zunächst beschrieben haben, geben wir nun einen Überblick über die in den Interaktionsverläufen entstehenden Muster in Abhängigkeit des Kontexts der Kita sowie des Geschlechts von Kinderbetreuenden und Kindern. 


\subsection{7 Überblick zur Verteilung der Interaktionsverläufe}

Die Tab. 4.3 gibt einen Überblick über die Kategorien in Bezug auf die Kitas, in Bezug auf das Geschlecht der Kinderbetreuenden und in Bezug auf das Geschlecht der beteiligten Kinder.

Die Ergebnisse zeigen, dass insgesamt diejenigen Interaktionsverläufe häufiger sind, bei denen die Kinderbetreuenden das Verhalten der Kinder verstärken, sei es in der Verstärkung des geschlechteruntypischen Verhaltens (Kind undoing gender, Betreuende Dramatisierung mit undoing gender, 40 Sequenzen) oder in der Verstärkung des geschlechterstereotypischen Verhaltens (Kind doing gender, Betreuende Dramatisierung mit doing gender, 34 Sequenzen). Wenn die Interaktionsverläufe nach dem Geschlecht des beteiligten Kindes analysiert werden, zeigt sich, dass mehr Interaktionen der Mädchen mit einem undoing gender beginnen, während die Interaktionen der Jungen etwas häufiger mit einem doing gender ihren Anfang nehmen. In Bezug auf das Geschlecht der Kinderbetreuenden zeigt sich, dass Männer einen etwas höheren Anteil für den Interaktionsverlauf undoing gender, Dramatisierung, undoing gender aufweisen. Diese Ergebnisse können jedoch nicht als Schlussfolgerungen über Unterschiede im Verhalten von Jungen und Mädchen, Männern und Frauen im Beruf der Kinderbetreuung interpretiert werden, die Datenbasis ist zu gering.

Die Verteilung in Tab. 4.3 zeigt zudem, dass es Unterschiede zwischen den untersuchten Kitas gibt. Die Abb. 4.2 visualisiert die unterschiedlichen Muster.

Während die in Kita Gelb, Grün und Blau analysierten Interaktionen alle einen ähnlichen Verlauf zeigen, mit den beiden Spitzen bei der Verstärkung von doing gender wie auch von undoing gender, zeigt sich bei Kita Rot ein anderes Bild. Es ist hier die Verstärkung von doing gender sehr wenig ausgeprägt, hingegen die Verstärkung von undoing gender viel mehr als in den anderen Kitas. Diese unterschiedlichen Muster wie auch unsere ethnografischen Beobachtungen legen nahe, dass in den Interaktionen im pädagogischen Alltag der Kitas Unterschiede in den pädagogischen Konzepten vorliegen müssen, die sich auch in der Organisationskultur niederschlagen.

\subsection{Analyse von (un)doing gender in pädagogischen Interaktionen mit Einbezug von Dramatisierung und Dethematisierung}

Im Folgenden werden zunächst die forschungsmethodische Vorgehensweise in Hinblick auf ihre Beschränkungen reflektiert sowie die Herausforderungen und 


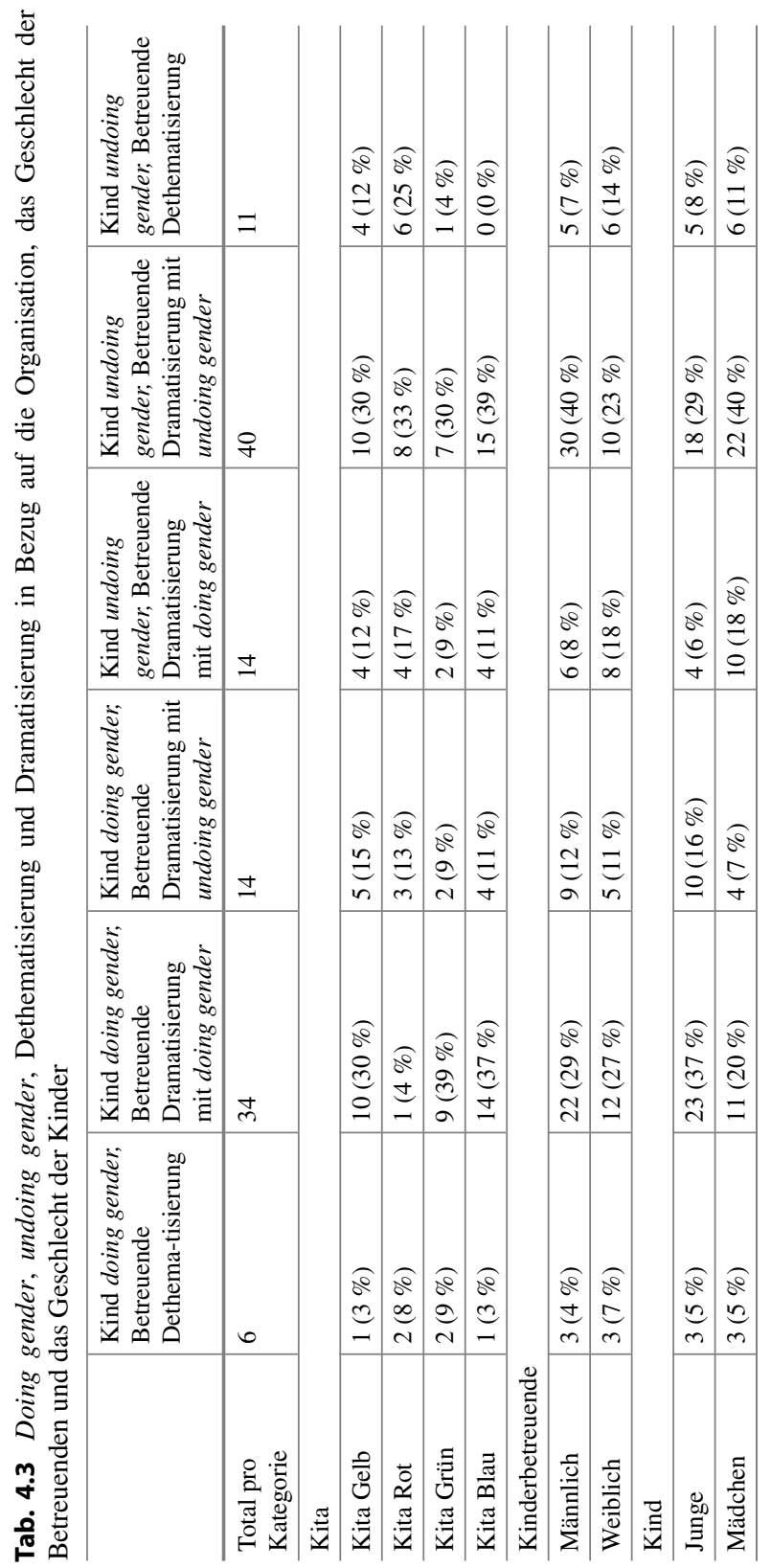




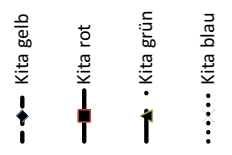

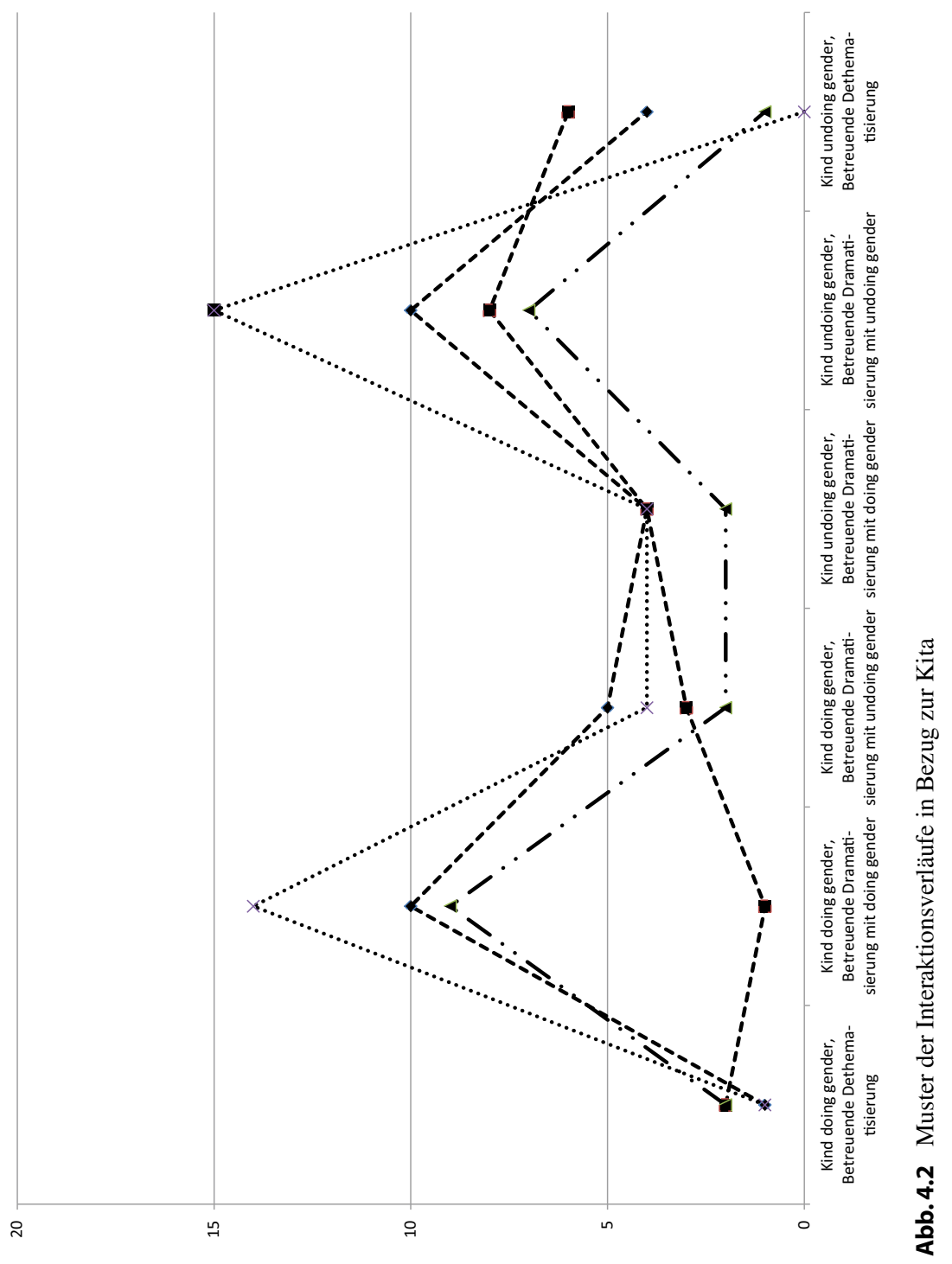


Chancen in theoretischer Sicht beleuchtet. Die Diskussion schließt mit Überlegungen zur Relevanz für die Praxis.

Für die Untersuchung der Interaktionen zwischen Kinderbetreuenden und Kindern in Bezug auf doing und undoing gender wurde die videogestützte Ethnografie genutzt. Die Videoaufnahmen waren eingebettet in die ethnografische Forschungsstrategie, es fanden zuerst Beobachtungen und Gespräche statt, sodass die Anwesenheit der Forschenden alltäglicher wurde. Trotzdem ist die Situation durch die Aufnahme, die Präsenz der Kameras und Knopfmikrofone ungewohnt, was die Interaktionen beeinflussen kann (Reh 2012). Das gesamte Videomaterial wurde gesichtet und mit den ethnografischen Notizen verglichen. Es wurden Sequenzen ausgewählt, in denen sich doing gender oder undoing gender zeigen, und Sequenzen, die für die jeweilige Alltagspraxis der Kita passend sind. In der Intercodierung innerhalb des Forschungsteams bestand diesbezüglich eine gute Übereinstimmung. Es ist jedoch eine der Beschränkungen des Vorgehens mit Event-Sampling, dass die Auswahl der Sequenzen für den Datenkorpus die nachfolgende Analyse beeinflusst.

Die hier analysierten Interaktionsverläufe beginnen mit einer Aktivität oder Aussage des Kindes und umfassen die darauffolgende Reaktion der Kinderbetreuenden. Der weitere Verlauf wird nicht mehr analysiert. Die Kinder interpretieren die Reaktion möglicherweise aufs Neue, widersprechen oder erleben eine Verstärkung ihres Tuns. Interaktionen und Interpretationen können zu einem anderen Zeitpunkt wieder aufgegriffen und umgedeutet werden. Insofern wird mit der hier vorgestellten Analyse eine Beschreibung der Mikroanalyse von Interaktionen vorgelegt, die jedoch die längerfristigen Verläufe nicht beachtet. Als weitere Einschränkung ist zu erwähnen, dass das hier gewählte Vorgehen keine Einblicke in die Überlegungen und Überzeugungen der Kinderbetreuenden für die gezeigte Reaktion gibt (Sandström et al. 2013). Dafür wäre eine Erweiterung des Designs in Richtung ,stimulated recall“ geeignet (Messmer 2015).

Die Analyse der Interaktionsverläufe ermöglicht hingegen, doing und undoing gender sowie Dramatisierung und Dethematisierung in den Alltagspraktiken der Kita zu fassen und damit die Fluidität (Nentwich und Kelan 2014) und das ständige Wechseln des Relevantwerdens von gender in der Kita zu berücksichtigen. Durch die Analyse der beiden Dimensionen doing/undoing gender und Dramatisierung/Dethematisierung gelingt es, diese Wechsel präziser zu fassen, die situative Realisierung Schritt für Schritt nachzuvollziehen und damit die aus der Interaktion entstehenden Verläufe dieser kurzen Sequenzen auf der Mikroebene zu fassen. Die Analyse ermöglicht einen Einblick darin, wie das Tun von gender in der Alltagspraxis vor sich geht, wie Geschlecht als kulturelle Ressource von 
den Kindern und Kinderbetreuenden genutzt oder ruhen gelassen, verstärkt oder unterlaufen wird.

Die detaillierte Analyse der Interaktionsverläufe zwischen Kindern und Kinderbetreuenden zeigt auf, wie Geschlecht als kulturelle Ressource in den pädagogischen Alltagsinteraktionen in der Kita relevant gemacht wird. Insbesondere die Verwendung der verschiedenen Perspektiven auf (un)doing gender hat sich für die Analyse der Interaktionsverläufe als fruchtbar erwiesen. Während die Konzeption Deutschs, zwischen doing und undoing anhand der Betonung von Differenz oder Gleichheit zu unterscheiden, ermöglichte, einzelne Handlungen zuzuweisen, erlaubte uns die Analyse der Dramatisierung und Dethematisierung, die Dynamik der Interaktion einzubeziehen. Für die Analyse im Forschungsprojekt ist diese klare Zuordnung von doing und undoing gender auf der Mikroebene des Interaktionsverlaufs sinnvoll, auch wenn dabei die in den Geschlechtsstereotypen verankerten und allgemein geteilten Wissensbestände durch die Zuweisung fortgeführt werden, was als Reifikation kritisiert werden könnte. Nur durch die sehr detaillierte Analyse der Interaktionen, die mehrere Schritte im Verlauf auseinanderhält und die Interpretationen expliziert, können die Geschlechterkonstruktionen und Umdeutungen im Kontext der Kita in den Blick genommen werden.

Die Unterscheidung zwischen dem Verhalten der Kinder und der Reaktion der Kinderbetreuenden im Interaktionsverlauf gibt beispielhaft Einblick, wie Kinderbetreuerinnen und Kinderbetreuer mit ihren situativen Reaktionen auf die Nutzung der kulturellen Ressource Geschlecht durch die Kinder diesen wiederum verschiedene Spielarten von Geschlecht vermitteln. Doing und undoing gender werden subtil im Alltag verstärkt oder aber nicht beachtet. Es kann vermutet werden, dass die Kinder auch auf diese Art und Weise die Normativität der Geschlechterordnung erfahren, ohne dass diese mit Sanktionen durchgesetzt werden würde (Maihofer 2002). Welche Wirkungen doing und undoing gender sowie Dramatisierungen und Dethematisierungen im pädagogischen Handeln der Kinderbetreuenden auf die Kinder haben, kann in dieser Studie nicht empirisch analysiert werden. Überlegungen darüber, welche Wirkungen dieses Verhalten hat, stützen sich auf theoretische Grundlagen.

Im Sinn der Förderung der Gleichstellung werten wir es als positiv, dass die Kinder nicht explizit für geschlechteruntypisches Verhalten mit Hinweisen wie „du bist ein Junge du solltest x tun“ oder ,ein Mädchen tut y nicht“ kritisiert werden. Auch reagieren die Kinderbetreuenden auf undoing gender häufig bestärkend mit Dramatisierung und undoing gender und weniger häufig durch Dramatisierung und doing gender. Dies könnte als Beitrag zur Förderung der Gleichstellung der 
Geschlechter gewertet werden, da eindeutige Zuordnungen von Verhaltensweisen oder Eigenschaften nicht klar einem bestimmten Geschlecht zugewiesen werden.

Insgesamt zeichnet sich bei drei der Kitas das Muster der Interaktionen dadurch aus, dass das, was die Kinder einbringen, sei es doing oder undoing gender, aufgenommen und verstärkt wird. Weniger häufig wird dem doing gender eines Kindes ein undoing gender in der Reaktion der Kinderbetreuenden entgegengesetzt. Dies steht möglicherweise in Zusammenhang mit den pädagogischen Überzeugungen der Kinderbetreuenden. Die Betonung des freien Spiels kann auch dazu führen, dass eine aktive Beteiligung der Kinderbetreuenden als negativ angesehen wird und Gesprächsanlässe, die das Relevantmachen von Gleichstellung im Alltag bieten würden, nicht genutzt werden. Dass sich die Kinderbetreuenden stark daran orientieren, was die Kinder einbringen und dies verstärken, sei es nun den geschlechtsstereotypen Erwartungen entsprechend oder aber widersprechend, lässt die Vermutung zu, dass es für eine geschlechterreflektierende Praxis alltagsnahe Trainings und Reflexion bräuchte, die zudem auf ihre Wirkung hin untersucht werden müssten. Wissenschaftliche Interventionsstudien zur Wirkung einer geschlechterreflektierenden Praxis in der frühen Kindheit fehlen jedoch bisher.

Die Muster der Interaktionsverläufe unterscheiden sich zwischen den Kitas. Während in drei Kitas jeweils das von den Kindern Eingebrachte verstärkt wird, scheint in Kita Rot ein deutlicherer Schwerpunkt auf der Verstärkung des undoing gender zu liegen. Das hier vorliegende Muster zeigt ein weniger häufiges Verstärken von doing gender, bei einer häufigeren Verstärkung von undoing gender der Kinder. Auch zeigen die Kinder in Kita Rot insgesamt weniger doing gender, d. h. sie engagieren sich weniger in geschlechterstereotypen Aktivitäten. Möglicherweise liegt hier eine Wechselwirkung vor und die Kinder fühlen sich in Kita Rot stärker ermutigt, nicht geschlechterstereotyp zu spielen. Auch in anderen Bereichen unserer Analyse zeigt sich, dass Kita Rot eher ein undoing gender ermöglicht und doing gender abschwächt. In der Analyse der Räume fiel auf, dass sich Kita Rot in der Raumgestaltung von anderen Kitas unterscheidet: Kita Rot hat keine räumlich getrennten Bauecken und Puppenstuben eingerichtet, die Verkleidungen bieten weiblich und männlich konnotierte Rollen an und die Kita legt Wert auf unstrukturiertes Material und Materialien zum Forschen und Entdecken. Es ist darum auch denkbar, dass diese geschlechterreflektierende Gestaltung der Räume dazu führt, dass die Kinder insgesamt weniger geschlechterstereotypische Spielaktivitäten wählen und wiederum weniger Interaktionen erfolgen, die von einem doing gender des Kindes ausgehen, das dann mit einer Dramatisierung der Kinderbetreuenden beantwortet werden könnte. Dass unterschiedliche Muster der Interaktionsverläufe zwischen Kita Rot und den anderen Kitas möglich 
sind, deutet auf die Bedeutung der pädagogischen Konzepte und deren organisationskultureller Umsetzung für die geschlechterreflektierende Praxis in Kitas hin.

In der Videoanalyse der Interaktionen wurde deutlich, dass die Kinder sowohl doing als auch undoing gender zeigen; die Kinderbetreuenden reagieren darauf mit Dramatisierung oder Dethematisierung. Insgesamt verstärken sie jedoch häufiger das von den Kindern ausgehende Verhalten. Eine geschlechterreflektierende Praxis könnte Kinderbetreuende motivieren, vermehrt darauf zu achten, doing gender nicht $\mathrm{zu}$ dramatisieren, sondern zu dethematisieren, und undoing gender entweder zu bestärken oder nicht zu beachten; wichtig ist auch, auf nicht geschlechterstereotype Handlungen, also auf undoing gender, nicht dramatisierend zu reagieren und mit doing gender ein geschlechterstereotypenkonformes Verhalten einzufordern. Die hier vorgelegte Mikroanalyse der Interaktionsverläufe wurde von der Autorin im Kontext von Weiterbildungen eingesetzt. Kinderbetreuende und weitere Pädagoginnen und Pädagogen nutzen sie zur gemeinsamen Reflexion der pädagogischen Praxis. Die Pädagoginnen und Pädagogen bringen eine Beobachtung aus dem Kita-Alltag ein, sie beschreiben die Handlung oder das Verhalten eines Kindes sowie die Reaktion der Kinderbetreuenden. Gemeinsam wird herausgearbeitet, wie die Interaktion dem Verlaufsschema gemäß anders verlaufen könnte, wie dadurch doing und undoing gender sowie Dramatisierung und Dethematisierung anders verlaufen. Als Beispiel sei die Situation mit dem Jungen und den Stöckelschuhen (Kind undoing gender) weitergedacht: Der Kinderbetreuer könnte nichts dazu sagen (Dethematisierung). Er könnte eine Dramatisierung und undoing gender machen, in dem er beispielsweise sagt: „Toll, wenn du lernst, mit Stöckelschuhen zu gehen!“‘. Die Reflexion über Varianten der Interaktionsverläufe macht es für die Kinderbetreuenden nachvollziehbarer, wie fluide und situativ doing gender und undoing gender geschieht und wie Interaktionen Geschlechterstereotype stärken oder schwächen können. Indem das Schema der Interaktionsverläufe die Wahl zwischen doing und undoing gender sowie zwischen Dramatisierung und Dethematisierung bewusst macht, zeigt es den pädagogischen Handlungsspielraum für eine geschlechterreflektierende Praxis auf.

\section{Literatur}

Brandes, Holger, Andrä, Markus, Röseler, Wenke, \& Schneider-Andrich, Petra (2013). Männer in Kitas - Was machen sie anders und wie profitieren die Kinder von ihnen? Ergebnisse 
aus der „Tandem-Studie“ zu professionellem Erziehungsverhalten von Männern und Frauen. Frühe Kindheit, 16(5), 38-43

Breidenstein, Georg, \& Kelle, Helga (2003). Mädchen und Jungen und Kinder - Kontexte der Geschlechterunterscheidung in der Schule. Weinheim: Beltz.

Butler, Judith (2004). Undoing Gender. Routledge: London.

Chick, Kay A., Heilman-Houser, Rose Anne, \& Hunter, Maxwell W. (2002). The Impact of Child Care on Gender Role Development and Gender Stereotypes. Early Childhood Education Journal, 29(3), 149-154.

Deutsch, Francine M. (2007). Undoing Gender. Gender \& Society, 21(1), 106-127.

Egert, Franziska, \& Dederer, Verena (2018). Metaanalyse zur Wirkung von Weiterbildungen für pädagogische Fachkräfte zur Steigerung der Interaktionsqualität. München: Staatsinstitut für Frühpädagogik. Verfügbar unter: https://www.ifp.bayern.de/imperia/md/con tent/stmas/ifp/metaanalyse_interaktionsqualitat_egert_dederer_2018_final.pdf.

Faulstich-Wieland, Hannelore, Weber, Martina \& Willems, Katharina (2004). Doing gender im heutigen Schulalltag. Empirische Studien zur sozialen Konstruktion von Geschlecht in schulischen Interaktionen. Weinheim: Juventa.

Gildemeister, Regine, \& Robert, Günther (2008). Geschlechterdifferenzierungen in lebenszeitlicher Perspektive: Interaktion-Institution-Biografie. Wiesbaden: VS Verlag für Sozialwissenschaften.

Goffman, Erving (1994). Das Arrangement der Geschlechter. In Hubert A. Knoblauch (Hrsg.), Interaktion und Geschlecht (S. 105-158). Frankfurt: Campus Verlag.

Gramespacher, Elke, \& Voss, Anja (2019). Geschlecht in der frühkindlichen Bewegungsbildung-eine vernachlässigte Dimension! Frühe Bildung, 8(2), 117-119

Gieß-Stüber, Petra, Voss, Anja \& Petry, Karen (2003). GenderKids - Geschlechteralltag in der frühkindlichen Bewegungsförderung. In Ilse Hartmann-Tews, Petra Gieß-Stüber, MarieLouise Klein, Christa Kleindienst-Cachay, \& Karen Petry (Hrsg.), Soziale Konstruktion von Geschlecht im Sport (S. 69-108). Opladen: Leske + Budrich.

Hyun, Eunsook, \& Choi, Dong Haw (2004). Examination of Young Children's Gender-Doing and Gender-Bending in their Play Dynamics: A Cross-Cultural Exploration. International Journal of Early Childhood, 36(1), 48-64.

Hirschauer, Stefan. (1994). Die soziale Fortpflanzung der Zweigeschlechtlichkeit. Kölner Zeitschrift für Soziologie und Sozialpsychologie, 46(4), 668-692.

Hollander, Jocelyn A. (2013). "I Demand More of People" Accountability, Interaction, and Gender Change. Gender \& Society, 27(1), 5-29.

Kelan, Elisabeth K. (2010). Gender Logic and (Un)doing Gender at Work. Gender, Work and Organization, 17(2), 174-194.

Kubandt, Melanie (2020). Die Vielfalt und Varianz geschlechtlicher Praktiken. Zum Mehrwert ethnographischer Forschungszugänge in der Kindertageseinrichtung. Diskurs Kindheitsund Jugendforschung, 15(1), 5-20.

Maihofer, Andrea (2002). Geschlecht und Sozialisation. Eine Problemskizze. Erwägen, Wissen, Ethik, EWE (vormals EuS), 13(1), 13-26.

Messmer, Roland (2015). Stimulated Recall als fokussierter Zugang zu Handlungs- und Denkprozessen von Lehrpersonen. Forum Qualitative Sozialforschung, 16(1).

Mortensen, Jennifer A., \& Barnett, Melissa A. (2015). Teacher-Child Interactions in Infant/Toddler Child Care and Socioemotional Development. Early Education and Development, 26(2). 
Nentwich, Julia C., \& Kelan, Elisabeth K. (2014). Towards a Topology of 'Doing Gender': An Analysis of Empirical Research and its Challenges. Gender, Work and Organization, 21(2), 121-134.

NICHD Early Child Care Research Network (2002). Child-Care Structure - Process - Outcome: Direct and Indirect Effects of Child Care Quality on Young Children's Development. Psychological Science, 13, 199-206.

Ostrov, Jamie M. \& Hart, Emily J. (2013). Observational Methods. In Todd D. Little (ed.), The Oxford Handbook of Quantitative Methods. Vol. 1: Foundations (S. 285-303). Oxford: Oxford University Press.

Papadopoulou, Marianna (2012). The Ecology of Role Play: Intentionality and Cultural Evolution. British Educational Research Journal, 38(4), 575-592.

Perren, Sonja, Frei, Doris, \& Herrmann, Sandra (2016). Pädagogische Qualität in frühkindlichen Bildungs- und Betreuungseinrichtungen in der Schweiz. Erste Erfahrungen und Befunde mit dem CLASS Toddler Beobachtungsverfahren. Frühe Bildung, 5(1), 3-12.

Pickering, Michael (2001). Stereotyping: The Politics of Representation. New York: Palgrave. Rabe-Kleberg, Ursula (2003). Gender Mainstreaming und Kindergarten. Weinheim: Beltz.

Reh, Sabine (2012). Mit der Videokamera beobachten. In Heike de Boer \& Sabine Reh (Hrsg.), Beobachtung in der Schule - Beobachten lernen (S. 151-169). Wiesbaden: VS Verlag für Sozialwissenschaften.

Rendtorff, Barbara (2011). Was Kinder spielen, wenn sie spielen - geschlechtstypische Aspekte im kindlichen Spiel. In Marcel Klaas, Alexandra Flügel, Rebecca Hoffmann, \& Bernadette Bernasconi (Hrsg.), Kinderkultur(en) (S. 71-83). Wiesbaden: VS Verlag für Sozialwissenschaften.

Sandström, Margareta, Stier, Jonas \& Sandberg, Annette (2013). Working with Gender Pedagogics at 14 Swedish Preschools. Journal of Early Childhood Research, 11(2), 123-132.

Suchodoletz, Antje von, Fäsche, Anika, Gunzenhauser, Catherine, \& Hamre, Bridget K. (2014). A Typical Morning in Preschool: Observations of Teacher-Child Interactions in German Preschools. Early Childhood Research Quarterly, 29(4), 509-519.

West, Candace, \& Zimmerman, Don H. (2009). Accounting for Doing Gender. Gender and Society, 23(1), 112-122.

Westheuser, Linus (2015). Männer, Frauen und Stefan Hirschauer. Undoing gender zwischen Praxeologie und rhetorischer Modernisierung. GENDER-Zeitschrift für Geschlecht, Kultur und Gesellschaft, 7(3), 109-125. 
Open Access Dieses Kapitel wird unter der Creative Commons Namensnennung 4.0 International Lizenz (http://creativecommons.org/licenses/by/4.0/deed.de) veröffentlicht, welche die Nutzung, Vervielfältigung, Bearbeitung, Verbreitung und Wiedergabe in jeglichem Medium und Format erlaubt, sofern Sie den/die ursprünglichen Autor(en) und die Quelle ordnungsgemäß nennen, einen Link zur Creative Commons Lizenz beifügen und angeben, ob Änderungen vorgenommen wurden.

Die in diesem Kapitel enthaltenen Bilder und sonstiges Drittmaterial unterliegen ebenfalls der genannten Creative Commons Lizenz, sofern sich aus der Abbildungslegende nichts anderes ergibt. Sofern das betreffende Material nicht unter der genannten Creative Commons Lizenz steht und die betreffende Handlung nicht nach gesetzlichen Vorschriften erlaubt ist, ist für die oben aufgeführten Weiterverwendungen des Materials die Einwilligung des jeweiligen Rechteinhabers einzuholen. 